\title{
Extensively drug-resistant Haemophilus influenzae - emergence, epidemiology, risk factors, and regimen
}

\author{
Pei-Yi Su', Ay-Huey Huang ${ }^{1}$, Chung-Hsu Lai ${ }^{2,3}$, Hsiu-Fang Lin ${ }^{1}$, Tsun-Mei Lin ${ }^{4}$ and Cheng-Hsun Ho ${ }^{4 *}$
}

\begin{abstract}
Background: Concern about Haemophilus influenzae infection has been increasing over recent decades. Given the emergence of $\mathrm{H}$. influenzae with severe drug resistance, we assessed the prevalence of as well as risk factors and potential therapies for extensively drug-resistant (XDR) H. influenzae infection in Taiwan.

Results: In total, 2091 H. influenzae isolates with disk diffusion-based antibiotic susceptibility testing from 2007 to 2018 were enrolled. H. influenzae strains resistant to ampicillin, chloramphenicol, levofloxacin, and trimethoprimsulfamethoxazole tended to be isolated from patient wards ( $\geqq 41 \%$ ), whereas those resistant to amoxicillinclavulanate, cefotaxime, and cefuroxime were more likely to be isolated from intensive care units (approximately 50\%). XDR H. influenzae was first identified in 2007, and its incidence did not significantly change thereafter. Overall prevalence of single, multiple, and extensively drug-resistant $H$. influenzae over 2007-2018 was $21.5 \%(n=450)$, $26.6 \%(n=557)$, and $2.5 \%(n=52)$, respectively. A stepwise logistic regression analysis revealed that blood culture (odds ratio: 4.069, 95\% confidence intervals: 1.339-12.365, $P=0.013$ ) was an independent risk factor for XDR $H$. influenzae infection. No nosocomial transmission of XDR H. influenzae observed. Antibiotic susceptibility testing results demonstrated that cefotaxime was effective against 78.8\% $(n=41)$ of the XDR strains.
\end{abstract}

Conclusions: The presence of XDR H. influenzae strains was identified in Taiwan, and cefotaxime was efficacious against most of these strains.

Keywords: Haemophilus influenzae, Antimicrobial susceptibility testing, Drug resistance, Cefotaxime

\section{Background}

Haemophilus influenzae is an opportunistic pathogen that can cause infections with various clinical symptoms, including otitis media, epiglottitis, sinusitis, and pneumonia, particularly in children, the elderly, and immunocompromised patients [1]. Transmission of $H$. influenzae occurs primarily through direct contact with respiratory droplets from pharyngeal carriers. Neonates may acquire infection by aspiration of amniotic fluids or by contact

\footnotetext{
* Correspondence: chenghsunho@gmail.com

${ }^{4}$ Department of Medical Laboratory Science, College of Medicine, I-Shou University, No.8, Yida Road, Jiaosu Village, Yanchao District, Kaohsiung City 82445, Taiwan

Full list of author information is available at the end of the article
}

with genital tract secretions containing living bacteria [2]. Without early and effective treatments, $H$. influenzae infection may result in life-threatening complications, such as bacteremia and meningitis, particularly in those infected with type b strains. Bacteremia can lead to amputation of limbs. Moreover, up to $30 \%$ of adult patients who survive meningitis suffer permanent hearing loss or other long-term neurological sequelae [3]. Approximately $5 \%$ of invasive $H$. influenzae infections in children are fatal.

Although the vaccination plan against the type $\mathrm{b}$ strain has been promoted globally, $H$. influenzae remains a formidable pathogen in Taiwan because of the late introduction of the vaccine, an unsatisfactory implementation

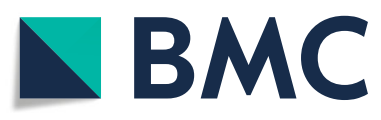

(c) The Author(s). 2020 Open Access This article is licensed under a Creative Commons Attribution 4.0 International License, which permits use, sharing, adaptation, distribution and reproduction in any medium or format, as long as you give appropriate credit to the original author(s) and the source, provide a link to the Creative Commons licence, and indicate if changes were made. The images or other third party material in this article are included in the article's Creative Commons licence, unless indicated otherwise in a credit line to the material. If material is not included in the article's Creative Commons licence and your intended use is not permitted by statutory regulation or exceeds the permitted use, you will need to obtain permission directly from the copyright holder. To view a copy of this licence, visit http://creativecommons.org/licenses/by/4.0/ The Creative Commons Public Domain Dedication waiver (http://creativecommons.org/publicdomain/zero/1.0/) applies to the data made available in this article, unless otherwise stated in a credit line to the data. 
rate, and infections caused by non-type b or nontypeable strains. With regard to treatment, ampicillin and first-line cephalosporins are becoming increasingly less efficacious against $H$. influenzae due to the development of various drug resistant mechanisms, such as $\beta$-lactamase activity and modified bacterial penicillin-binding proteins [4-6]. Second or third-generation cephalosporins or quinolones have become more favorable options for the treatment of $\mathrm{H}$. influenzae infection [7]. However, physicians should more cautiously select antibiotics because of the thriving $H$. influenzae that is non-susceptible to amoxicillin-clavulanate, fluoroquinolones, second or third-generation cephalosporins, and macrolides [7-12]; hence, carbapenems or combination therapies might be empirically considered if appropriate.

Multiple drug-resistant (MDR) H. influenzae, which is generally defined as non-susceptibility to at least one agent in three or more antimicrobial categories [13], was first reported in West Germany in 1980 [14]. In that report, two strains were non-susceptible to ampicillin, chloramphenicol, and tetracycline. The emergence of MDR $H$. influenzae has aroused the widespread concern of government health agencies, medical communities, and researchers worldwide. Much effort has been taken to reveal risk factors, adequate management control, prevention, and underlying mechanisms of acquired MDR activity [15-17]. Despite growing evidence of the identification of MDR $H$. influenzae strains in different countries [18-21], no study has demonstrated extensively drug-resistant (XDR) H. influenzae, defined as non-susceptibility to at least one agent in all but two or fewer antimicrobial categories [13]. Herein, we reported the emergence of XDR $H$. influenzae in South Taiwan. Furthermore, risk factors and antimicrobial susceptibilities of XDR $H$. influenzae were characterized.

\section{Results}

Epidemiology of drug non-susceptibility in $\mathrm{H}$. influenzae Characteristics of the patients and specimens $(n=2091)$ from which $H$. influenzae isolates were obtained are shown in Table 1 . Most of the specimens were taken from respiratory tracts $(n=1915 ; 91.6 \%)$ including sputa, bronchial brushings, bronchial washings, and bronchoalveolar lavages. Forty-five $(2.1 \%)$ isolates were obtained from blood cultures and 131 (6.3\%) from wounds, abscesses, or body fluids. More than half of the $H$. influenzae isolates were non-susceptible to ampicillin or trimethoprim-sulfamethoxazole (Fig. 1a). In addition, 7.6, 2.7 , and $14.1 \%$ of $H$. influenzae isolates were nonsusceptible to cefuroxime, cefotaxime, and levofloxacin, respectively. Remarkably high resistance rates of $H$. influenzae to ampicillin was recorded in 2007 (69.6\%) and to amoxicillin-clavulanate in 2007 (27.7\%) and 2017 (34.0\%). The non-susceptible rate of $H$. influenzae to chloramphenicol or cephems was less than $30 \%$, except for chloramphenicol in 2015 and cefuroxime in 2017. Trends of drug non-susceptibility in different types of specimens are shown in Additional file 1: Figure S1. $\beta$ lactamase activity was screened in $933 \mathrm{H}$. influenzae isolates and significantly increased the drug non-susceptibility of ampicillin $(P=0.05)$ but not other antimicrobial agents (Fig. 1b). The demographics of the patients had almost no effect on the drug susceptibility of $H$. influenzae (Additional file 2: Figure S2).

\section{Drug non-susceptibility of specimens from different origins}

$H$. influenzae strains that were isolated from specimens collected in the emergency room had the lowest nonsusceptibility frequency to antimicrobial agents, except

Table 1 Characteristics of patients and specimens with Haemophilus influenzae

\begin{tabular}{|c|c|c|c|c|c|}
\hline Year & Number & Patient gender (male: female) & Patient age & Specimen type (Respiratory, blood, others) & Specimen source (OPD, ER, Wards, ICUs) \\
\hline 2007 & 184 & 115: 69 & $75.7 \pm 18.6$ & $173,5,6$ & $14,8,102,60$ \\
\hline 2008 & 279 & 192: 87 & $73.0 \pm 19.3$ & $262,3,14$ & $23,23,159,74$ \\
\hline 2009 & 203 & 143: 60 & $68.0 \pm 21.5$ & $188,2,13$ & $19,21,106,57$ \\
\hline 2010 & 149 & 104: 45 & $69.4 \pm 21.5$ & $133,1,15$ & $26,20,62,41$ \\
\hline 2011 & 271 & 187: 84 & $63.8 \pm 24.4$ & $218,6,47$ & $77,42,96,56$ \\
\hline 2012 & 183 & 135: 48 & $64.2 \pm 21.9$ & $146,2,35$ & $38,36,75,34$ \\
\hline 2013 & 159 & 96: 63 & $61.7 \pm 22.3$ & $134,6,19$ & $48,33,45,33$ \\
\hline 2014 & 170 & 126: 44 & $66.0 \pm 21.0$ & $144,5,21$ & $39,32,69,30$ \\
\hline 2015 & 123 & 88: 35 & $67.0 \pm 20.4$ & $109,3,11$ & $21,18,54,30$ \\
\hline 2016 & 154 & $97: 57$ & $62.8 \pm 19.0$ & $125,5,24$ & $48,13,62,13$ \\
\hline 2017 & 97 & 69: 28 & $61.7 \pm 18.4$ & $86,3,8$ & $18,8,40,31$ \\
\hline 2018 & 119 & 84: 35 & $60.9 \pm 21.5$ & $99,4,16$ & $36,11,42,30$ \\
\hline Total & 2091 & 1436: 655 & $66.8 \pm 21.5$ & $1915,45,131$ & $407,265,912,507$ \\
\hline
\end{tabular}

Age is shown as mean \pm standard deviation. Specimens other than those from respiratory tracts and blood include wound, pus, abscess, body fluids, and tissues. Abbreviations: $E R$ emergency room, ICU intensive care unit, OPD outpatient department 
a

All specimens $(n=2091)$

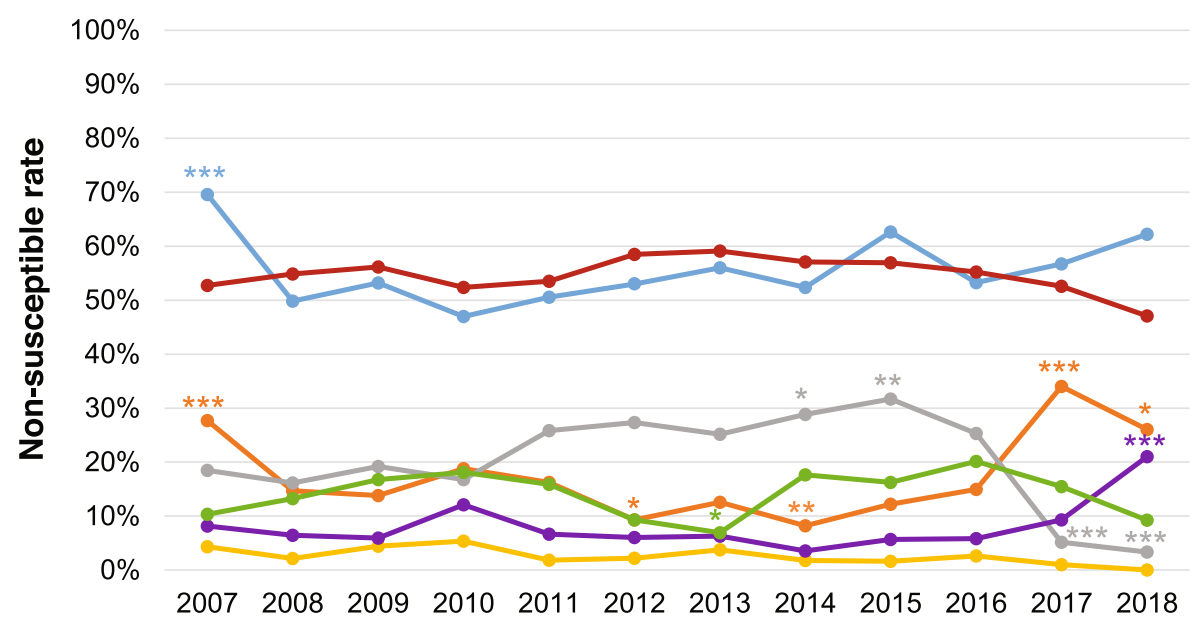

$\rightarrow$ Ampicillin $(\bar{x}=54.9 \%)$

$\rightarrow$ Amoxicillin-clavulanate $(\bar{x}=16.5 \%)$

$\rightarrow$ Chloramphenicol $(\bar{x}=21.0 \%) \multimap$ Cefotaxime $(\bar{x}=2.7 \%)$

$\rightarrow$ Cefuroxime $(\bar{x}=7.6 \%) \quad \rightarrow$ Levofloxacin $(\bar{x}=14.1 \%)$

$\rightarrow$ Trimethoprim-sulfamethoxazole $(\bar{x}=54.9 \%)$

b

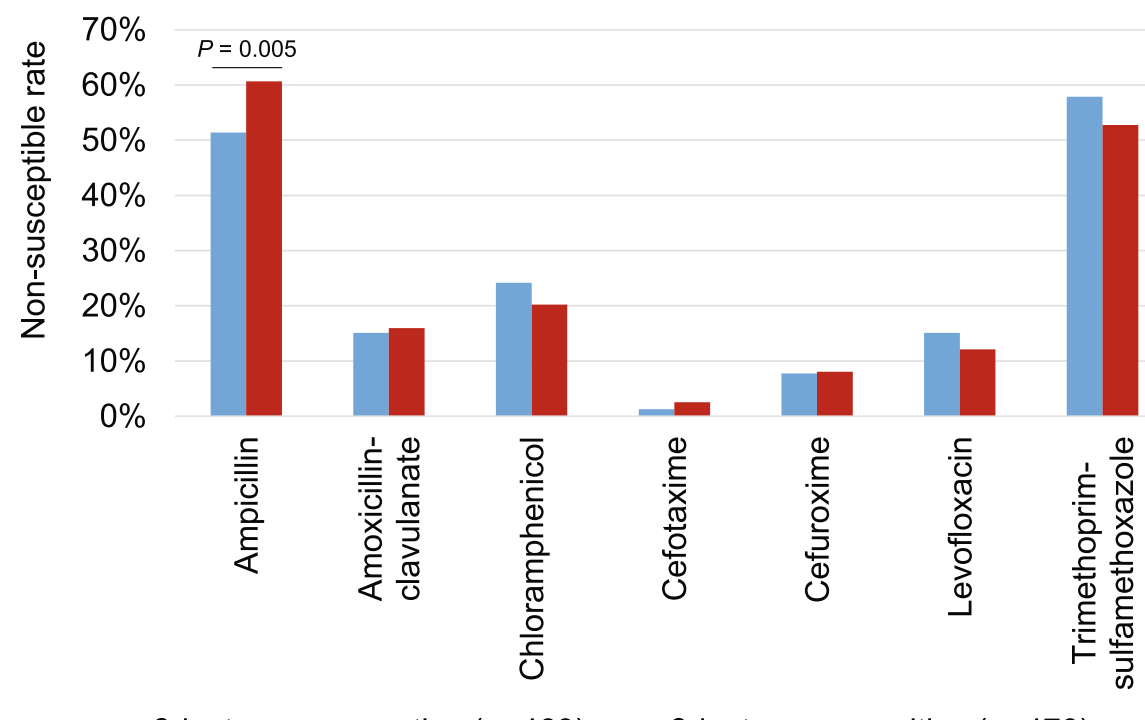

- $\beta$-lactamase negative $(n=463) \quad$ - $\beta$-lactamase positive $(n=470)$

Fig. 1 Drug non-susceptibility in Haemophilus influenzae. a Incidences of drug non-susceptibility to different antimicrobial agents in $H$. influenzae isolates from 2007 to $2018(n=2091)$ are shown. Fisher's exact tests or Pearson Chi-square tests were used to assess the significance of the nonsusceptible rate of each drug in each year when compared to the overall mean value. ${ }^{*} P<0.05$, ${ }^{* *} P<0.01$, ${ }^{* * *} P<0.001 . \bar{x}$, mean value. $\mathbf{b}$

Comparisons of drug non-susceptibility rates in $\beta$-lactamase-positive and $\beta$-lactamase-negative $H$. influenzae isolates. $P$-values are obtained from Fisher's exact tests

chloramphenicol (Fig. 2a). Patient wards were the major origin of $H$. influenzae isolates that were non-susceptible to ampicillin, chloramphenicol, levofloxacin, and trimethoprim-sulfamethoxazole, whereas intensive care units were the main origin of isolates non-susceptible to amoxicillin-clavulanate, cefuroxime, and cefotaxime. Notably, $H$. influenzae strains isolated from respiratory care wards or respiratory care centers were more susceptible to amoxicillin-clavulanate, cefotaxime, and cefuroxime but more often non-susceptible to chloramphenicol 
a

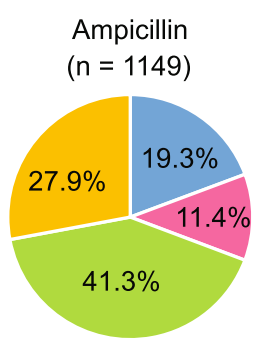

Amoxicillin-clavulanate

$(n=345)$

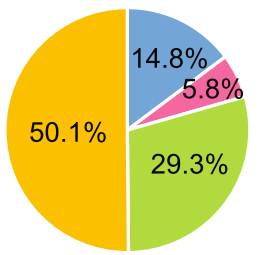

Cefuroxime

$(n=158)$

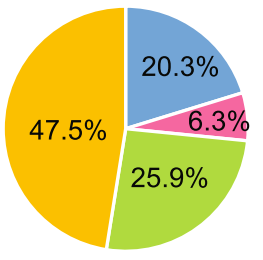

$(n=295)$

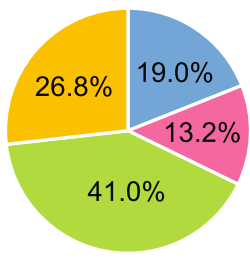

Drug non-susceptibility

Chloramphenicol

$(n=439)$
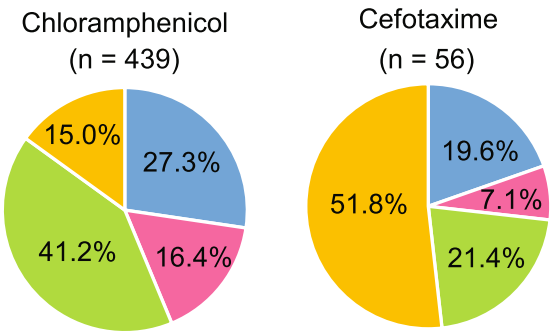

Trimethoprimsulfamethoxazole $(n=1147)$
Outpatient department

Patient wards

Intensive care units
Emergency room

b

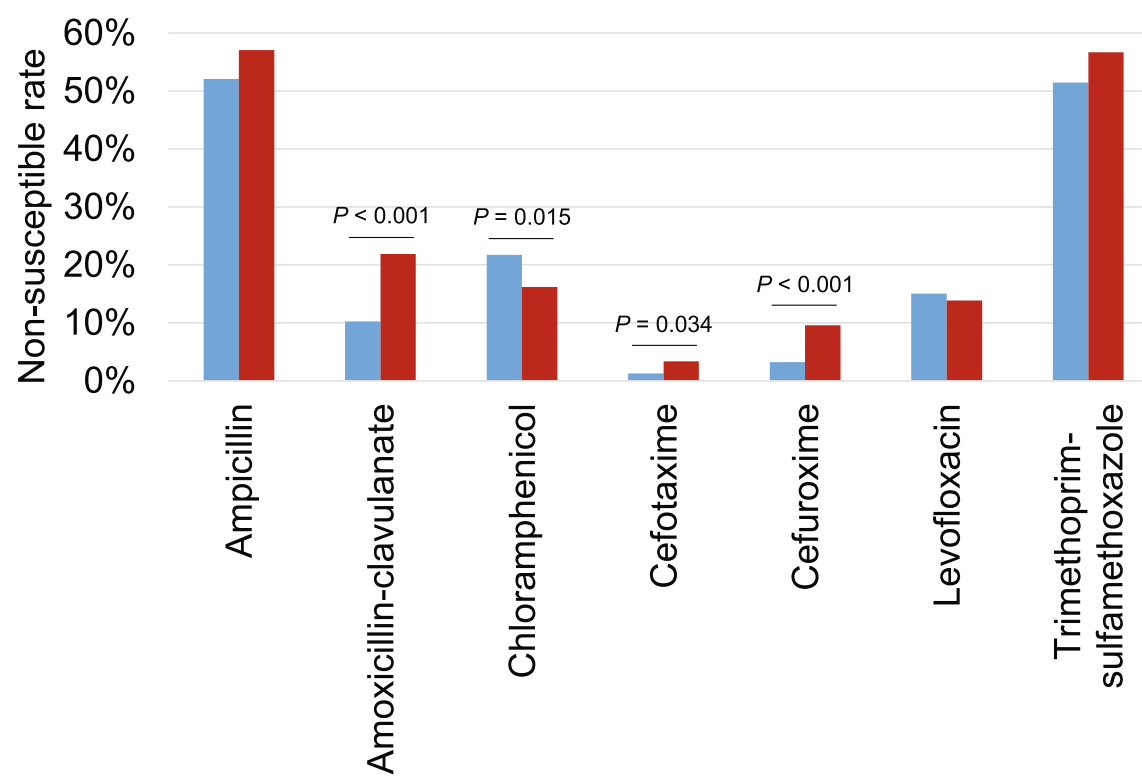

$\square \operatorname{RCW} / \operatorname{RCC}(\mathrm{n}=313) \quad$ Other wards/ICUs $(\mathrm{n}=1106)$

Fig. 2 Drug non-susceptibility of Haemophilus influenzae from different origins. a Percentages of drug non-susceptible H. influenzae in specimens from different departments are shown. $\mathbf{b}$ Comparisons of drug non-susceptibility rates in hospitalized patients between respiratory care wards/ center $(n=313)$ and other units $(n=1106)$ are shown in column graphs. $P$-values are obtained from Chi-square tests

compared with other wards or intensive care units (Fig. 2b).

\section{MDR and XDR H. influenzae}

XDR $H$. influenzae strains were isolated from samples collected as far back as 2007. Incidences of single-drug resistance, MDR, and XDR H. influenzae from 2007 to
2018 were $21.5 \%$ (450/2091), $26.6 \%$ (557/2091), and $2.5 \%$ (52/2091), respectively (Fig. 3a). The drug resistance status remained consistent year by year (Fig. 3a, $P=0.526$ ) and was not affected by the demography of the patients (Additional file 3: Figure S3A). Overall, MDR strains ranged from approximately 15 to $30 \%$ and XDR strains were all below $5 \%$. $\beta$-lactamase activity did not correlate 


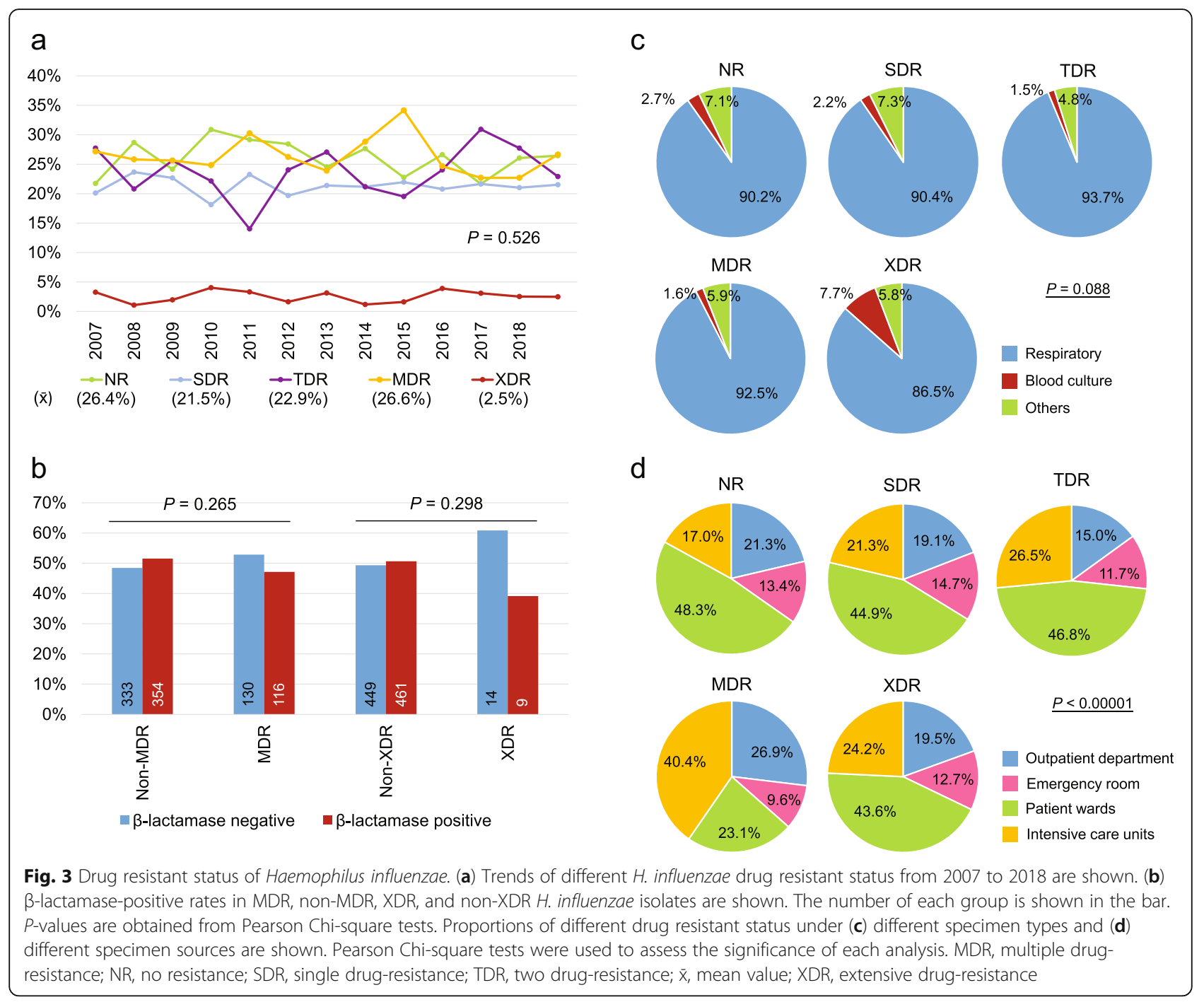

with the incidence of MDR $(P=0.265)$ or XDR $(P=$ 0.298) $H$. influenzae (Fig. 3b). The drug nonsusceptibility panels of MDR and XDR $H$. influenzae strains are shown in Table 2.

The type of specimen was not associated with the drug resistant status of $H$. influenzae (Fig. 3c). However, 7.7\% of XDR strains were isolated from blood cultures. Regarding the origin of the specimen, MDR strains were more likely to be isolated from intensive care units (Fig. 3d). Neither MDR nor XDR $H$. influenzae strains were linked to hospital-acquired infections (Additional file 3: Figure S3B). Characteristics of the patients and specimens with XDR $H$. influenzae are shown in Additional file 4: Table S1. Among these 52 XDR $H$. influenzae strains, six were susceptible to chloramphenicol only and three to levofloxacin only (Table 3 ). Moreover, one strain was susceptible to cefuroxime and chloramphenicol and one was susceptible to cefuroxime and levofloxacin. The other 41 strains (78.8\%) were either susceptible to cefotaxime only or cefotaxime together with an additional antimicrobial agent. These results suggest that cefotaxime is a potent antimicrobial agent for the management of XDR H. influenzae infection.

\section{Clinical relevance of MDR and XDR $H$. influenzae}

Stepwise logistic regression analyses revealed that MDR $H$. influenzae strains were less likely to be isolated from male patients (odds ratio [OR]: 0.769, 95\% confidence intervals [CI]: 0.624-0.946; $P=0.013$ ) (Table 4). Furthermore, a relationship between MDR $H$. influenzae and intensive care units (OR: 1.410, 95\% CI: 1.094-1.818; $P=0.008)$ was noted. XDR H. influenzae was more likely to be isolated from blood cultures (OR: 4.069, 95\% CI: $1.339-12.365 ; P=0.013)$. $\beta$-lactamase activity was not associated with MDR (OR: 0.985, 95\% CI: 0.875-1.109; $P=0.802$ ) or XDR (OR: $0.936,95 \%$ CI: $0.671-1.304 ; P=$ 0.694) H. influenzae. Furthermore, MDR (OR: 1.408, 95\% CI: $0.789-1.392 ; P=0.747)$ and XDR (OR: 0.394, 
Table 2 Panels of drug non-susceptibility in multiple drug resistant (MDR) and extensive drug resistant (XDR) Haemophilus influenzae isolates

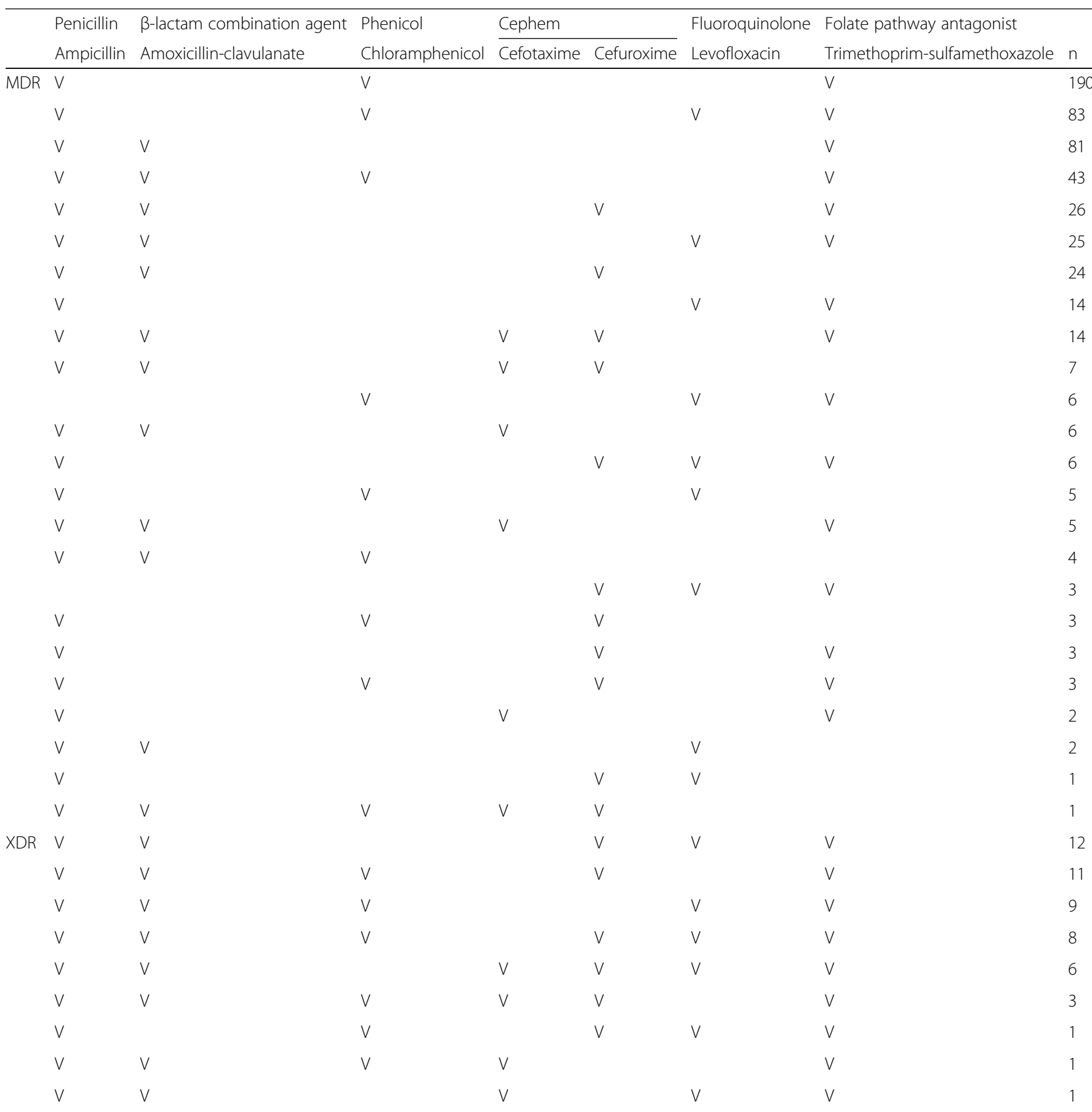

Multiple drug resistant (MDR) is defined as acquired non-susceptibility to at least one agent in three of five antimicrobial categories or non-susceptibility to at least one agent in four of five antimicrobial categories but less than five antimicrobial agents. Extensive drug resistant (XDR) is defined as acquired nonsusceptibility to at least one agent in four of five antimicrobial categories and at least five antimicrobial agents

95\% CI: 0.122-1.274; $P=0.120) H$. influenzae strains were not transmitted via hospital-acquired modes of infection.

\section{Discussion}

A high prevalence of certain drug-resistant bacterial species, promoted by antibiotic overuse within medical communities and patient therapeutic incompliance, has been noticed in Taiwan [12, 22-24]. MDR and XDR are often used to refer to Staphylococcus aureus, Enterococcus species, Enterobacteriaceae (other than Salmonella and Shigella), Pseudomonas aeruginosa, and Acinetobacter species because of their epidemiological significance, emerging antimicrobial resistance, and the importance of these bacteria within the healthcare system [13]. MDR $H$. influenzae is an aspect that is rarely 
Table 3 Cross table of antimicrobial agent susceptibility in 52 extensive drug resistant Haemophilus influenzae isolates

\begin{tabular}{|c|c|c|c|c|c|c|c|}
\hline & $\mathrm{AM}$ & AMC & C & CTX & CXM & LVX & SXT \\
\hline$A M$ & $0(0.0 \%)$ & & & & & & \\
\hline AMC & $0(0.0 \%)$ & $0(0.0 \%)$ & & & & & \\
\hline C & $0(0.0 \%)$ & $0(0.0 \%)$ & $6(11.5 \%)$ & & & & \\
\hline CTX & $0(0.0 \%)$ & 1 (1.9\%) & 12 (23.1\%) & 8 (15.4\%) & & & \\
\hline CXM & $0(0.0 \%)$ & $0(0.0 \%)$ & 1 (1.9\%) & 9 (17.3\%) & 0 (0.0\%) & & \\
\hline LVX & $0(0.0 \%)$ & $0(0.0 \%)$ & $0(0.0 \%)$ & $11(21.2 \%)$ & 1 (1.9\%) & $3(5.8 \%)$ & \\
\hline SXT & $0(0.0 \%)$ & $0(0.0 \%)$ & $0(0.0 \%)$ & $0(0.0 \%)$ & $0(0.0 \%)$ & $0(0.0 \%)$ & $0(0.0 \%$ \\
\hline
\end{tabular}

Data are shown as numbers (\%). AM ampicillin, $A M C$ amoxicillin-clavulanate, $C$ chloramphenicol, $C T X$ cefotaxime, CXM cefuroxime, LVX levofloxacin, SXT trimethoprim-sulfamethoxazole

discussed, and XDR H. influenzae has never been mentioned. Here we report the presence of MDR and XDR $H$. influenzae in Taiwan and address their clinical risk factors.

Although the Taiwanese government introduced selfpay $H$. influenzae type b strain vaccination in 2005 and launched nationwide vaccination in infants from 2010, $H$. influenzae remains a critical problem for people without immunization or with invasive infections caused by non-type $\mathrm{b}$ or non-typeable strains, which are now the most common causes of invasive $H$. influenzae infection in many countries [25-27]. One of the limitations of the present study is that serotyping of the $H$. influen$z a e$ isolates was not available because most of these bacterial isolates were not preserved until the recent approval by the biosafety committee.

Table 4 Logistic regression analyses of factors associated with multiple drug resistance or extensive drug resistance in Haemophilus influenzae

\begin{tabular}{|c|c|c|c|c|c|c|c|c|}
\hline \multirow[t]{3}{*}{ Variable } & \multicolumn{4}{|c|}{ Multiple drug resistant } & \multicolumn{4}{|c|}{ Extensive drug resistant } \\
\hline & \multicolumn{2}{|l|}{ Univariate } & \multicolumn{2}{|l|}{ Multivariate } & \multicolumn{2}{|l|}{ Univariate } & \multicolumn{2}{|l|}{ Multivariate } \\
\hline & $\begin{array}{l}\text { Odds ratio }(95 \% \\
\text { Cl) }\end{array}$ & $P$ & $\begin{array}{l}\text { Odds ratio }(95 \% \\
\text { Cl) }\end{array}$ & $P$ & Odds ratio $(95 \% \mathrm{Cl})$ & $P$ & Odds ratio $(95 \% \mathrm{Cl})$ & $P$ \\
\hline \multicolumn{9}{|l|}{ Demography } \\
\hline Year & $0.995(0.966-1.025)$ & 0.735 & & & $1.019(0.938-1.106)$ & 0.664 & & \\
\hline Age & $0.997(0.993-1.002)$ & 0.244 & & & $0.994(0.982-1.006)$ & 0.332 & & \\
\hline Gender $($ Male $=1$, Female =0) & $0.786(0.640-0.966)$ & 0.022 & $0.769(0.624-0.946)$ & 0.013 & $1.128(0.615-2.071)$ & 0.697 & & \\
\hline \multicolumn{9}{|l|}{ Specimen type } \\
\hline $\begin{array}{l}\text { Respiratory tracts }(\mathrm{Yes}=1, \mathrm{No}= \\
\text { 0) }\end{array}$ & $1.174(0.818-1.684)$ & 0.385 & & & $0.581(0.258-1.308)$ & 0.190 & & \\
\hline Blood (Yes $=1, \mathrm{No}=0$ ) & $0.683(0.327-1.428)$ & 0.311 & & & $4.061(1.399-11.790)$ & 0.010 & $4.069(1.339-12.365)$ & 0.013 \\
\hline Others $($ Yes $=1, \mathrm{No}=0)$ & $0.923(0.614-1.386)$ & 0.699 & & & $0.914(0.281-2.973)$ & 0.881 & & \\
\hline $\begin{array}{l}\text { B-lactamase (Positive }=1 \\
\text { Negative } \quad=0)^{\mathrm{a}}\end{array}$ & $0.985(0.875-1.109)$ & 0.802 & & & $0.936(0.671-1.304)$ & 0.694 & & \\
\hline \multicolumn{9}{|l|}{ Specimen source } \\
\hline $\begin{array}{l}\text { Outpatient department (Yes }=1 \text {, } \\
\text { No }=0 \text { ) }\end{array}$ & $1.141(0.897-1.451)$ & 0.284 & & & $1.543(0.828-2.876)$ & 0.172 & & \\
\hline $\begin{array}{l}\text { Emergency room (Yes }=1, \mathrm{No}= \\
\text { 0) }\end{array}$ & $0.861(0.638-1.162)$ & 0.327 & & & $0.728(0.287-1.847)$ & 0.504 & & \\
\hline Wards (Yes $=1, \mathrm{No}=0)$ & $0.695(0.570-0.849)$ & $\begin{array}{l}< \\
0.001\end{array}$ & $0.816(0.648-1.029)$ & 0.086 & $0.380(0.198-0.728)$ & 0.004 & $0.517(0.245-1.091)$ & 0.083 \\
\hline $\begin{array}{l}\text { Intensive care units (Yes }=1 \text {, } \\
\text { No }=0 \text { ) }\end{array}$ & $1.541(1.240-1.915)$ & $\begin{array}{l}< \\
0.001\end{array}$ & $1.410(1.094-1.818)$ & 0.008 & $2.165(1.233-3.802)$ & 0.007 & $1.695(0.880-3.265)$ & 0.115 \\
\hline \multicolumn{9}{|l|}{ Infection route } \\
\hline $\begin{array}{l}\text { Hospital-acquired infection } \\
(\text { Yes }=1, \mathrm{No}=0)\end{array}$ & $1.048(0.789-1.392)$ & 0.747 & & & $0.394(0.122-1.274)$ & 0.120 & & \\
\hline
\end{tabular}

Multiple drug resistance is defined as acquired non-susceptibility to at least one agent in three or more antimicrobial categories. Extensive drug resistance is defined as susceptible to only one antimicrobial category. Specimens other than those from respiratory tracts and blood include wound, pus, abscess, body fluids, and tissues. Hospital-acquired infection is defined as an infection occurs after 7 days of hospital admission. ${ }^{a} n=933$. Abbreviation: $C l$ confidence intervals 
The treatment of $H$. influenzae infection relies mainly on $\beta$-lactam antibiotics, predominantly ampicillin. However, modifications of penicillin-binding proteins and the spread of plasmids carrying $\beta$-lactamase genes, e.g. TEM-1 [28] and ROB-1 [29], among H. influenzae or by other bacterial species, have made ampicillin or other first-line $\beta$-lactam antibiotics inefficacious against $H$. influenzae. Not surprisingly, the epidemiology shows that rates of $H$. influenzae that were non-susceptible to ampicillin and amoxicillin-clavulanate were high, especially in 2007. A later decline in ampicillin or amoxicillin-clavulanate non-susceptibility might result from the more prudent use of antibiotics [30], a decrease in antibiotic prescribing by general practices [31], or preference for other classes of antimicrobial agents, such as cephalosporins and quinolones. There has been little information on $H$. influenzae strains that are nonsusceptible to cefotaxime. One multicenter study by Wang et al. demonstrated that $5.9 \%$ of $H$. influenzae isolates from children in China were non-susceptible to cefotaxime [11]. An Iranian meta-analysis of a collection of 43 articles from different databases showed that the prevalence of $H$. influenzae that was non-susceptible to cefotaxime was 22.3\% [32]. Cefotaxime resistance in H. influenzae is primarily caused by amino acid substitutions N526K, S385T, and L389F and additional substitutions G555E and Y557H in penicillin-binding protein 3 [10, 33, 34]. Our survey shows that the prevalence of cefotaxime-non-susceptible $H$. influen$z a e$ in Taiwan from 2007 to 2018 (2.7\%) is not as high as those in the above-mentioned reports. Nevertheless, unlike the sporadic cases reported in other countries [35-38], levofloxacin resistance in $H$. influenzae in Taiwan is more severe. A 6-year multicenter study revealed that the nonsusceptibility rate of $H$. influenzae to levofloxacin in Taiwan is $12.5 \%$, and all resistant isolates had at least three mutations in the quinolone resistance-determining regions of GyrA and $\operatorname{ParC}$ [9]. The good news is that the incidence of levofloxacin-non-susceptible $H$. influenzae reduced from $20.1 \%$ in 2016 to $9.2 \%$ in 2018.

In our center, $73.2 \%(41 / 56)$ of cefotaxime nonsusceptible isolates and $67.8 \%(200 / 295)$ of levofloxacin non-susceptible isolates came from hospitalized patients. Interestingly, $H$. influenzae isolated from respiratory care wards or respiratory care centers were more susceptible to amoxicillin-clavulanate, cefuroxime, and cefotaxime. This could be attributed to the much less exposure to these three drugs owing to different antibiotics being preferred for the relief of severe respiratory infections caused by other bacterial pathogens. Nonetheless, the link between intensive care units and MDR strains shows that enhanced alertness should be exercised in the treatment of patients with severe infections such as meningitis or septicemia that are caused by $H$. influenzae infection.
According to the guideline from the Clinical \& Laboratory Standards Institute, azithromycin, clarithromycin, tetracycline, ertapenem, and imipenem are listed as group $\mathrm{C}$ antimicrobial agents for $\mathrm{H}$. influenzae. Macrolides, tetracycline, or carbapenems are not the primary choice of treatment for $H$. influenzae infection in most medical care institutions in Taiwan, including our center. Therefore they are not being included in our routine drug susceptibility test unless required by special medical circumstances. Our survey provided valuable information despite not including all categories of antimicrobial agents. Among the 557 MDR H. influenzae strains, the most common drug non-susceptibility panel was ampicillin, chloramphenicol and trimethoprimsulfamethoxazole $(n=190,34.1 \%)$ and the second most common panel was ampicillin, chloramphenicol, levofloxacin and trimethoprim-sulfamethoxazole $(n=83$, 14.9\%). For XDR $H$. influenzae strains, the most common drug non-susceptibility panel was ampicillin, amoxicillin-clavulanate, cefuroxime, levofloxacin and trimethoprim-sulfamethoxazole $(n=12,23.1 \%)$. Of note, eight XDR strains were non-susceptible to all six drug categories tested, suggesting that complex drug-resistant mechanisms might be involved in the development of severely drug-resistant $H$. influenzae. Blood culture was an independent factor for the isolation of XDR H. influenzae. More studies are needed to elucidate whether these XDR strains are inherently invasive and then acquire resistance mechanisms or whether XDR confers an invasive ability to these strains.

The outpatient department, which is a pivotal index for monitoring household and community transmissions, contributes to nearly $20 \%$ of the XDR $H$. influenzae strains as well. The Division of Infectious Disease and the Antibiotic Stewardship at our center established standard operating procedures for the management of severe drug resistant bacteria; therefore all patients with XDR $H$. influenzae in our study had received proper medication and healthcare and exhibited no signs of relapse. There are currently no signs of XDR $H$. influenzae nosocomial infection or group infection. We will continue to track the incidence of XDR H. influenzae.

\section{Conclusion}

This study reported the emergence, epidemiology, risk factors, and treatment regimen of XDR $H$. influenzae infection. The mean incidence of XDR $H$. influenzae infection from 2007 to 2018 was approximately $2.5 \%$. Fortunately, a group infection or nosocomial spread of XDR $H$. influenzae has not been identified yet. Drug susceptibility testing reveals that cefotaxime still has an efficacy against about $80 \%$ of XDR $H$. influenzae strains. Greater attention from a public health point of view should be paid to this problem. Additional prophylactic 
medication strategies are required to prevent the development and spread of XDR H. influenzae infection.

\section{Methods}

\section{Study design}

This study was approved by the Institutional Review Board (EMRP-106-062) and performed at E-DA Hospital (Kaohsiung, Taiwan). Data of 2411 laboratory identifications of $H$. influenzae were collected from 2007 to 2018. After excluding related tests from the same patients during a medication management course, $2091 \mathrm{H}$. influenzae isolates from 1436 male and 655 female patients were finally enrolled. Hospital-acquired infection is defined as infection that occurs after 7 days of hospital admission. Twenty-four patients had received the vaccination for infants against the type b strain of $H$. influenzae. Other patients had no anamnesis of $H$. influenzae vaccination. All the patients were treated according to the antibiotic stewardship of E-DA Hospital.

\section{Laboratory identification and drug-susceptibility tests}

$H$. influenzae was identified using Oxoid $\mathrm{X}+\mathrm{V}$ factor Disc (Thermo Fisher Scientific, Waltham, MA, USA) prior to 2014 and mass spectrometry (VITEK MS, BioMérieux, Marcy-l'Étoile, France) after 2014. Antimicrobial susceptibility tests were performed using BBL Sensi-Disc antimicrobial susceptibility test discs (Becton, Dickinson and Company, Sparks, MD, USA). The following seven antimicrobial agents were included: ampicillin $(10 \mu \mathrm{g})$, amoxicillin-clavulanate $(20 / 10 \mu \mathrm{g}), \quad$ chloramphenicol $(30 \mu \mathrm{g})$, cefotaxime $(30 \mu \mathrm{g})$, cefuroxime $(30 \mu \mathrm{g})$, levofloxacin $(5 \mu \mathrm{g})$, and trimethoprim-sulfamethoxazole $(1.25 / 23.75 \mu \mathrm{g})$. Drug susceptibility was evaluated according to the guideline from the Clinical \& Laboratory Standards Institute. ETESTs (BioMérieux) were performed on 100 randomly selected $H$. influenzae isolates to confirm the drug susceptibility patterns. BBL Cefinase ${ }^{\mathrm{T \mu}}$ discs (Becton, Dickinson and Company) were used for the rapid detection of $\beta$-lactamase activity in $933 \mathrm{H}$. influenzae isolates.

\section{Definition of drug resistance status}

Six antimicrobial categories, including penicillin (ampicillin), $\beta$-lactam combination agent (amoxicillin-clavulanate), phenicol (chloramphenicol), cephem (cefotaxime and cefuroxime), fluoroquinolone (levofloxacin), and folate pathway antagonist (trimethoprim-sulfamethoxazole) were classified according to the guideline from the Clinical \& Laboratory Standards Institute (Additional file 5: Table S2). No drug resistance is defined as susceptibility to all antimicrobial agents. Single-drug resistance is defined as non-susceptibility to at least one agent in one antimicrobial category. Two-drug resistance is defined as non-susceptibility to at least one agent in two antimicrobial categories. MDR is defined as non-susceptibility to at least one agent in three or four antimicrobial categories. XDR is defined as non-susceptibility to at least one agent in five or six antimicrobial categories.

\section{Statistical analysis}

SPSS 18.0 for Windows was used for all statistical analyses. Nominal variables were compared using Fisher's exact tests or Pearson Chi-square tests. Continuous variables were compared using Student's $t$-tests for two independent groups and one-way analysis of variance with Scheffe's post hoc tests for multiple groups. For the time-point studies, the incidences of drug nonsusceptibility in each year were compared with the overall mean value. Stepwise logistic regression analyses were performed to evaluate factors that were associated with MDR or XDR $H$. influenzae. Significance is set at $P<0.05$ (2-tailed).

\section{Supplementary information}

Supplementary information accompanies this paper at https://doi.org/10 1186/s12866-020-01785-9.

Additional file 1 : Figure S1. Drug non-susceptibility in Haemophilus influenzae isolates from different specimen types.

Additional file $\mathbf{2}$ : Figure S2. Association of the demography of patients with drug non-susceptibility in Haemophilus influenzae isolates.

Additional file $\mathbf{3}$ : Figure S3. Association of the demography of patients and infection route with the drug resistant status of Haemophilus influenzae.

Additional file 4 : Table S1. Characteristics of the patients and specimens of extensive drug resistant Haemophilus influenzae isolates.

Additional file $\mathbf{5}$ : Table S2. Breakpoints used to determine susceptible, intermediate, and resistant categories for Haemophilus influenzae based on CLSI interpretative criteria.

Additional file 6. Dataset. Dataset used in this study.

\section{Abbreviations}

AM: ampicillin; AMC: amoxicillin-clavulanate; C: chloramphenicol; Cl: confidence intervals; CTX: cefotaxime; CXM: cefuroxime; ER: emergency room; H. influenzae: Haemophilus influenzae; ICU: intensive care unit; LVX: levofloxacin; MDR: multiple drug-resistance; OPD: outpatient department; OR: odds ratio; SXT: trimethoprim-sulfamethoxazole; XDR: extensive drug-resistance

\section{Acknowledgments}

We thank the Department of Information Technology of E-DA Hospital for providing demographic and clinical data of the patients. This manuscript was edited by Wallace Academic Editing by native speakers of English.

\section{Authors' contributions}

PYS was responsible for the study design and the interpretation of results of bacterial identification and drug susceptibility tests; AHH, HFL, and TML assisted in data collection. CHL assisted in the analysis of hospital-acquired infection. $\mathrm{CHH}$ was responsible for statistical analyses, manuscript writing, and composed the manuscript. All authors read and approved the final manuscript. PYS and $\mathrm{AHH}$ contributed equally to this paper.

\section{Funding}

This work was supported by grant EDAHT107006 from E-DA Hospital medical research project and MOST 108-2314-B-214-003-MY2 from the Ministry of Science and Technology, Taiwan. The funding body had the role of paying the consumption materials used in this study and had no role in the design 
of the study and collection, analysis, and interpretation of data and in writing the manuscript.

\section{Availability of data and materials}

The data in this study can be seen in Additional file 6: Dataset.

\section{Ethics approval and consent to participate}

This study was approved by the Institutional Review Board (EMRP-106-062) and performed at E-DA Hospital in accordance with the ethical standards noted in the 1964 Declaration of Helsinki and its later amendments or comparable ethical standards. Written informed consents were waived by the Institutional Review Board of E-DA Hospital because the study involved no more than minimal risk of harm to subjects.

\section{Consent for publication}

Not applicable.

\section{Competing interests}

All authors declared no conflict of interests.

\section{Author details}

'Department of Laboratory Medicine, E-DA Hospital, Kaohsiung, Taiwan. ${ }^{2}$ School of Medicine, College of Medicine, I-Shou University, Kaohsiung, Taiwan. ${ }^{3}$ Division of Infectious Diseases, Department of Internal Medicine, E-Da Hospital, Kaohsiung, Taiwan. ${ }^{4}$ Department of Medical Laboratory Science, College of Medicine, I-Shou University, No.8, Yida Road, Jiaosu Village, Yanchao District, Kaohsiung City 82445, Taiwan.

\section{Received: 1 October 2019 Accepted: 7 April 2020}

\section{Published online: 28 April 2020}

\section{References}

1. Tsang RSW, Ulanova M. The changing epidemiology of invasive Haemophilus influenzae disease: emergence and global presence of serotype a strains that may require a new vaccine for control. Vaccine. 2017;35:4270-5.

2. Cox RA, Slack MP. Clinical and microbiological features of Haemophilus influenzae vulvovaginitis in young girls. J Clin Pathol. 2002;55:961-4.

3. Agrawal A, Murphy TF. Haemophilus influenzae infections in the $H$. influenzae type b conjugate vaccine era. J Clin Microbiol. 2011;49:3728-32.

4. Nathwani D, Wood MJ. Penicillins. A current review of their clinical pharmacology and therapeutic use. Drugs. 1993;45:866-94.

5. Neu HC. The crisis in antibiotic resistance. Science. 1992;257:1064-73.

6. Low DE. Resistance trends in Haemophilus influenzae (PROTEKT years 1-3 [1999-20021). J Chemother 2004;16 Suppl 6:49-61.

7. Tristram S, Jacobs MR, Appelbaum PC. Antimicrobial resistance in Haemophilus influenzae. Clin Microbiol Rev. 2007;20:368-89.

8. Nazir J, Urban C, Mariano N, Burns J, Tommasulo B, Rosenberg C, et al. Quinolone-resistant Haemophilus influenzae in a long-term care facility: clinical and molecular epidemiology. Clin Infect Dis. 2004;38:1564-9.

9. Kuo SC, Chen PC, Shiau YR, Wang HY, Lai JFs, Huang W, et al. Levofloxacinresistant haemophilus influenzae, Taiwan, 2004-2010. Emerg Infect Dis 2014; 20:1386-1390.

10. Mizoguchi A, Hitomi S. Cefotaxime-non-susceptibility of Haemophilus influenzae induced by additional amino acid substitutions of G555E and Y557H in altered penicillin-binding protein 3. J Infect Chemother. 2019;25: 509-13.

11. Wang HJ, Wang CQ, Hua CZ, Yu H, Zhang T, Zhang H, et al. Antibiotic resistance profiles of Haemophilus influenzae isolates from children in 2016: a Multicenter study in China. Can J Infect Dis Med Microbiol. 2019;2019: 6456321.

12. Huang YT, Hsueh PR. Antimicrobial drug resistance in Taiwan. Int J Antimicrob Agents. 2008;32(Suppl 3):S174-8.

13. Magiorakos AP, Srinivasan A, Carey RB, Carmeli Y, Falagas ME, Giske CG, et al. Multidrug-resistant, extensively drug-resistant and pandrug-resistant bacteria: an international expert proposal for interim standard definitions for acquired resistance. Clin Microbiol Infect. 2012;18:268-81.

14. Braveny I, Machka K. Multiple resistant Haemophilus influenzae and parainfluenzae in West Germany. Lancet. 1980;2:752-3.

15. Campos J, Chanyangam M, de Groot R, Smith AL, Tenover FC, Reig R. Genetic relatedness of antibiotic resistance determinants in multiply resistant Hemophilus influenzae. J Infect Dis. 1989;160:810-7.
16. Dimopoulou ID, Kraak WA, Anderson EC, Nichols WW, Slack MP, Crook DW. Molecular epidemiology of unrelated clusters of multiresistant strains of Haemophilus influenzae. J Infect Dis. 1992;165:1069-75.

17. Levy J, Verhaegen G, De Mol P, Couturier M, Dekegel D, Butzler JP. Molecular characterization of resistance plasmids in epidemiologically unrelated strains of multiresistant Haemophilus influenzae. J Infect Dis. 1993;168:177-87.

18. Hansman D. Multiple drug resistance in Haemophilus influenzae type b. Med J Aust. 1985;142:536-7.

19. Pfeifer $Y$, Meisinger I, Brechtel K, Grobner S. Emergence of a multidrug-resistant Haemophilus influenzae strain causing chronic pneumonia in a patient with common variable immunodeficiency. Microb Drug Resist. 2013;19:1-5.

20. Tribuddharat C, Srifuengfung S. Multiple drug resistance in Haemophilus influenzae isolated from patients in Bangkok, Thailand. J Glob Antimicrob Resist. 2017;9:121-3.

21. Hara N, Wajima T, Seyama S, Tanaka E, Shirai A, Shibata M, et al. Isolation of multidrug-resistant Haemophilus influenzae harbouring multiple exogenous genes from a patient diagnosed with acute sinusitis. J Infect Chemother. 2019;25:385-7.

22. Hsueh PR, Luh KT. Antimicrobial resistance in Streptococcus pneumoniae, Taiwan. Emerg Infect Dis. 2002;8:1487-91.

23. Hsueh PR, Liu CY, Luh KT. Current status of antimicrobial resistance in Taiwan. Emerg Infect Dis. 2002;8:132-7.

24. Hsueh PR, Chen ML, Sun CC, Chen WH, Pan HJ, Yang LS, et al. Antimicrobial drug resistance in pathogens causing nosocomial infections at a university hospital in Taiwan, 1981-1999. Emerg Infect Dis. 2002;8:63-8.

25. Ladhani S, Slack MP, Heath PT, von Gottberg A, Chandra M, Ramsay ME. European Union invasive bacterial infection surveillance p. invasive Haemophilus influenzae disease, Europe, 1996-2006. Emerg Infect Dis. 2010;16:455-63.

26. Van Eldere J, Slack MP, Ladhani S, Cripps AW. Non-typeable Haemophilus influenzae, an under-recognised pathogen. Lancet Infect Dis. 2014;14:1281-92.

27. Slack MPE. The evidence for non-typeable Haemophilus influenzae as a causative agent of childhood pneumonia. Pneumonia (Nathan). 2017:9:9.

28. Medeiros AA, O'Brien TF. Ampicillin-resistant Haemophilus influenzae type B possessing a TEM-type beta-lactamase but little permeability barrier to ampicillin. Lancet. 1975;1:716-9.

29. Rubin LG, Medeiros AA, Yolken RH, Moxon ER. Ampicillin treatment failure of apparently beta-lactamase-negative Haemophilus influenzae type b meningitis due to novel beta-lactamase. Lancet. 1981;2:1008-10.

30. Lee $\mathrm{CR}$, Cho $\mathrm{H}$, Jeong BC, Lee $\mathrm{SH}$. Strategies to minimize antibiotic resistance. Int J Environ Res Public Health. 2013:10:4274-305.

31. Ranji SR, Steinman MA, Shojania KG, Gonzales R. Interventions to reduce unnecessary antibiotic prescribing: a systematic review and quantitative analysis. Med Care. 2008;46:847-62.

32. Vaez H, Sahebkar A, Pourfarzi F, Yousefi-Avarvand A, Khademi F. Prevalence of antibiotic resistance of Haemophilus Influenzae in Iran- a meta-analysis. Iran J Otorhinolaryngol. 2019;31:349-57.

33. Skaare D, Anthonisen IL, Kahlmeter G, Matuschek E, Natas OB, Steinbakk M, et al. Emergence of clonally related multidrug resistant Haemophilus influenzae with penicillin-binding protein 3-mediated resistance to extended-spectrum cephalosporins, Norway, 2006 to 2013. Euro Surveill. 2014;19:20986.

34. Sondergaard A, Norskov-Lauritsen N. Contribution of PBP3 substitutions and TEM-1, TEM-15, and ROB-1 Beta-lactamases to Cefotaxime resistance in Haemophilus influenzae and Haemophilus parainfluenzae. Microb Drug Resist. 2016;22:247-52.

35. Karlowsky JA, Thornsberry C, Critchley IA, Jones ME, Evangelista AT, Noel GJ, et al. Susceptibilities to levofloxacin in Streptococcus pneumoniae, Haemophilus influenzae, and Moraxella catarrhalis clinical isolates from children: results from 2000-2001 and 2001-2002 TRUST studies in the United States. Antimicrob Agents Chemother. 2003;47:1790-7.

36. Bastida T, Perez-Vazquez M, Campos J, Cortes-Lletget MC, Roman F, Tubau $F$, et al. Levofloxacin treatment failure in Haemophilus influenzae pneumonia. Emerg Infect Dis. 2003;9:1475-8.

37. Ho PL, Chow KH, Mak GC, Tsang KW, Lau YL, Ho AY, et al. Decreased levofloxacin susceptibility in Haemophilus influenzae in children, Hong Kong. Emerg Infect Dis. 2004;10:1960-2.

38. Kim IS, Lee NY, Kim S, Ki CS, Kim SH. Reduced levofloxacin susceptibility in clinical respiratory isolates of Haemophilus influenzae is not yet associated with mutations in the DNA gyrase and topoisomerase II genes in Korea. Yonsei Med J. 2011;52:188-91.

\section{Publisher's Note}

Springer Nature remains neutral with regard to jurisdictional claims in published maps and institutional affiliations. 